\title{
Políticas paradiplomáticas orientadas a las Cadenas Globales de Valor ${ }^{1}$
}

\author{
Paradiplomatic policies oriented towards Global \\ Value Chains
}

\author{
Santiago Andrés Calvo²
}

Resumen: En la actualidad se presenta incuestionable la estrecha relación entre las acciones locales y globales, principalmente cuando se habla de economía. Así, pues, resulta oportuno profundizar en la vinculación de dos áreas propias de la disciplina de las Relaciones Internacionales: las Cadenas Globales de Valor y la Paradiplomacia. Productos de las dinámicas globales vigentes, ambos fenómenos contribuyen a sostener la creciente participación de las relaciones económicas internacionales en los ámbitos locales. Por esta razón, este artículo pretende obtener un mayor entendimiento de los sistemas de producción deslocalizados pero claramente articulados bajo CGV, así como una nítida comprensión de las políticas paradiplomáticas. El propósito de este estudio es generar una incipiente variedad de política paradiplomática económica que permita a los actores subnacionales diseñar estrategias que maximicen la participación de empresas trasnacionales en sus territorios.

Palabras Clave: Políticas paradiplomáticas, Cadenas Globales de Valor, Paradiplomacia económica, upgrading.

Abstract: Currently, the close relationship between local and global actions is unquestionable, especially when it comes to economics. Thus, it seems appropriate to further research the connection between two areas within the field of International Relations: Global Value Chains and Paradiplomacy. Products of the current global dynamics, both phenomena contribute to sustaining the growing participation of international economic relations in local spheres. For this reason, this article aims to obtain a better understanding of delocalized but clearly articulated production systems under CGV, as well as a clear understanding of paradiplomatic policies. The purpose of this study is to create an incipient variety of economic paradiplomatic policy that allows subnational actors to design strategies that maximize the participation of transnational companies in their territories.

Key words: Paradiplomatic policies, Global Value Chains, Economic Paradiplomacy, upgrading.

\footnotetext{
DOI: $10.24215 / 23142766 \mathrm{e} 089$

${ }^{1}$ Recibido 25/09/2019. Aceptado 29/10/2019.

2 Becario doctoral de CONICET en el Instituto de Relaciones Internacionales (UNLP), Magíster en Relaciones Económicas Internacionales (UBA), Licenciado en Relaciones Internacionales y Licenciado en Ciencia Política (US21). Correo electrónico: lic.santiagocalvo@gmail.com
} 


\section{Introducción}

La progresiva vinculación entre las dinámicas locales y globales se asienta en una economía mundial altamente interconectada. En este escenario, se presenta oportuno profundizar el conocimiento de las relaciones internacionales y los espacios locales a partir de la vinculación de dos áreas propias de la disciplina: las Cadenas Globales de Valor y la Paradiplomacia. Ambas son consecuencias de las dinámicas globales vigentes que contribuyen a sostener la creciente participación de las relaciones económicas internacionales en los ámbitos locales.

En consecuencia, por un lado, observamos cómo las empresas transnacionales se convirtieron en el vehículo que coadyuvó de manera indiscutible al proceso de virtual desvanecimiento de las fronteras nacionales iniciado con la globalización, a partir de un proceso fragmentado de producción de bienes y servicios articulados entre sí, transformando la matriz de comercio e inversiones en un fenómeno sistémico e integrado.

Por otro, somos conscientes de que el debilitamiento de las fronteras no sólo vino de la mano del mundo empresarial; otros actores, diferentes al Estado central, comenzaron a tener una progresiva injerencia en asuntos internacionales, como es el caso de las unidades subnacionales. Su creciente protagonismo les permitió actuar como agentes de las relaciones internacionales es pos de sus propios intereses y como espacios articuladores de complejos productivos fraccionados globalmente.

La continua vinculación entre las dinámicas locales y globales se asienta en una economía mundial altamente interconectada, lo que confirma sin lugar a dudas el auge de la glocalización, concepto esbozado por Robertson décadas atrás. Por su parte, Hocking (2000, p. 28) hace referencia a ese término al observar una "relación simbiótica de fuerzas que cohesionan o que fragmentan, lo que constituye una dialéctica global-local, donde los sucesos locales se integran dentro de estructuras globales que, a su vez, inciden en los sucesos locales dentro de un continuum iterativo".

En definitiva, lo que se espera lograr con el presente artículo es comprender las dinámicas imperantes en las CGV, así como también los alcances de las políticas paradiplomáticas. Ello permitirá conjugarlas en un concepto preliminar de política paradiplomática orientada a maximizar los beneficios para los actores locales, en el marco de un consolidado proceso de división internacional de la producción. Para esto, en la primera sección se buscará extraer los principales elementos conceptuales de CGV, la ascendente importancia que ha adquirido dicha herramienta analítica y la distribución de los beneficios de formar en estas estructuras de producción globales.

En las siguientes secciones se estudiarán, en principio, los inicios y la consolidación del término paradiplomacia, y se distinguirán las diversas de acciones que los actores subnacionales pueden implementar. Luego, se analizarán las políticas paradiplomáticas económicas, en pos de avanzar en el estudio de acciones afines a la localización de factores productivos. Por último, se trabajará sobre un subtipo preliminar de política paradiplomática que incentive, no sólo la radicación de eslabones productivos de las CGV en territorios locales, sino también que estimule la superación de las barreras de entradas hacia eslabones más sofisticados y con mayores rentas. 


\section{Modelo de Producción Global: CGV}

A los fines del presente trabajo, se empleará el modelo de producción global denominado Cadena Global de Valor (CGV), el cual ha sido investigado por diversos autores y permite explicar el apogeo del comercio intraindustrial, el cual representa una proporción creciente de las exportaciones mundiales de los últimos años. Este modelo identifica un proceso de deslocalización geográfica de la producción a nivel planetario, el cual maximiza sus beneficios a partir de la radicación de diversas actividades en distintos puntos del globo. En palabras de Lall y otros (2004, p. 56), estamos en presencia de "sistemas internacionales establecidos para optimizar la producción, la comercialización y la innovación mediante la localización de productos, procesos o funciones en diferentes países para beneficiarse de los bajos costos, del marketing tecnológico, logístico y otras diferencias".

Una CGV describe toda la gama de actividades que las empresas realizan para llevar un producto, desde su concepción hasta su uso final. Esto incluye actividades tales como el diseño, la producción, la comercialización, la distribución y la asistencia al consumidor final. Las actividades que la integran pueden ser realizadas dentro de una misma empresa o divididas entre diferentes, las que a su vez pueden ser ejecutadas dentro de una única ubicación geográfica o estar distribuidas en diversas áreas del globo (The Global Value Chains -GVC- Initiative $)^{3}$.

La importancia de las CGV tomó protagonismo de la mano de organismos internacionales, como la Organización Mundial del Comercio (OMC), Organización para la Cooperación y el Desarrollo Económicos (OCDE), Conferencia de las Naciones Unidas sobre Comercio y Desarrollo (UNCTAD) y la Comisión Económica para América Latina y el Caribe (CEPAL), quienes han puesto su análisis en la agenda económica mundial. En los últimos años ${ }^{4}$, han elaborado una serie de documentos sobre el tema con el objetivo de medir su importancia en el comercio mundial.

Esta relevancia viene dada por una serie de aspectos, a saber: 1) su estrecha relación con la inversión extranjera directa (IED); 2) el progresivo incremento del intercambio de bienes intermedios; 3) el aumento del contenido importado de las exportaciones; 4) el papel fundamental que cumplen los servicios, muchos de los cuales se incorporan como insumos a los bienes finales comercializados; $y, 5$ ) la mayor agregación del valor radicada en las actividades intensivas en conocimiento como diseño e investigación (Oddone, 2016b).

De este modo, los organismos internacionales plantean una visión optimista de la globalización, las CGV y sus efectos potenciales sobre el desarrollo económico. Si bien su propuesta teórica no es objeto de análisis del presente trabajo, los supuestos que promulgan permiten tomarlos como un punto de referencia para un análisis integral de esta herramienta analítica. Los organismos internacionales presumen que la mera participación de los países en desarrollo en las cadenas globales de valor actuaría como un mecanismo de acceso

\footnotetext{
${ }^{3}$ The Global Value Chains Initiative es una red informal de investigadores, activistas y formuladores de políticas que busca consolidar y fomentar la perspectiva CGV, una visión centrada en la industria de la globalización económica que pone de relieve los vínculos entre los actores económicos y en todo el espacio geográfico. www.globalvaluechains.org
}

${ }^{4}$ OCDE y OMC, 2012, 2013; OCDE, 2013. 
a los mercados internacionales de bienes y servicios. Por tanto, los gobiernos no deben fomentar el establecimiento de una cadena de valor completa, sino participar en determinadas actividades y procesos. De esta manera, para poder beneficiarse plenamente de las CGV, sería necesario disminuir los costos de los insumos intermedios y servicios, liberar las inversiones, la propiedad intelectual e implementar políticas que faciliten el comercio.

En consecuencia, la integración en las CGV ofrecería una vía rápida hacia el desarrollo y la industrialización. Entre las ventajas que se pueden obtener de la participación en la transnacionalización de la producción, podemos hallar el fortalecimiento del tejido industrial, la atracción de nuevos capitales, la generación de empleo y el fomento de la productividad empresarial a partir de la adquisición de nuevas prácticas productivas y estándares tecnológicos -en consonancia con las prácticas internacionales-, entre otros. Todo ello contribuiría de manera positiva al fortalecimiento de la competitividad de los países.

A pesar de estos potenciales beneficios económicos, sus efectos distributivos y derrames sobre las economías domésticas no siempre son homogéneos. De acuerdo a Padilla (2014), "estos beneficios no son el producto espontáneo e inmediato del proceso de inserción en las cadenas (locales, regionales o globales), sino que requieren del adecuado funcionamiento de diversos factores como el sistema de relaciones de los actores, las instituciones de apoyo, los espacios de apropiación de valor agregado y, en general, de la gobernanza de la cadena."

La heterogeneidad en la distribución de los beneficios es sostenida por autores neoschumpeterianos, ${ }^{5}$ quienes plantean que no todas las actividades dentro de una CGV presentan las mismas ventajas para las economías nacionales. Así, algunas actividades de la parte superior de la cadena -diseño, investigación y desarrollo, producción de componentes avanzados-, y de la parte inferior de la cadena -marketing y distribución- tienden a generar mayor valor agregado que aquellas intermedias, por ejemplo, el ensamblado. Por tanto, la ubicación que tengan las empresas en las CGV determinará los beneficios para las economías nacionales.

Habiendo planteado las asimetrías entre las diversas actividades productivas deslocalizadas, el análisis de una cadena global de valor ayuda a entender de qué manera un país participa en una industria global, así como también cuál es su papel en la distribución de beneficios que se derivan de ella. La participación en distintos eslabones repercute de manera directa en la organización industrial de un país, así como también en su potencial de desarrollo económico y social, debido a que las actividades asociadas con cada eslabón emplean diversas combinaciones de recursos (humanos, naturales y capital). La profundización de los encadenamientos productivos existentes y el estímulo a la formación de nuevos, combinada con la incorporación de innovaciones tecnológicas, permite el escalamiento hacia actividades más complejas dentro de la cadena global de valor (CEPAL, 2013). Es por ello que los autores neo-schumpeterianos consideran al estudio de las CGV una herramienta conceptual indispensable para comprender las oportunidades de desarrollo de las economías menos avanzadas.

\footnotetext{
${ }^{5}$ Con el rótulo de "neo-schumpeterianos" se hace referencia a Gary Gereffi, Raphael Kaplinksy, Timothy Sturgeon y John Humphrey, entre otros.
} 
Aunque mencionado someramente, un concepto central dentro del modelo de CGV son las estructuras de gobierno de la cadena, es decir, las relaciones y mecanismos institucionales a través de los cuales se logra su coordinación. Así, el concepto de gobernanza tiene implicaciones que van más allá de la mera idea de cooperación, como es la existencia de una asimétrica distribución de poder dentro de la cadena. Generalmente, son grandes corporaciones las que asumen el liderazgo en la organización de la división del trabajo entre las empresas participantes, y ejercen un mayor o menor control sobre el desarrollo de las actividades, la estructura y la dinámica de la cadena. A su vez, este papel protagonista les permite retener una mayor porción del valor agregado total generado en la cadena.

La comprensión de la gestión de una cadena ayudará a vislumbrar la distribución de las ganancias a lo largo de ella. En relación con esto, Kaplinsky (2000) sugiere que la capacidad de gobernar a menudo se basa en las competencias intangibles ( $+D$, diseño, marca, marketing) que están caracterizadas por altas barreras de entrada y de altos rendimientos, generalmente obtenidas por las empresas de países desarrollados. Por el contrario, en los países en desarrollo las empresas tienden a ser bloqueadas en las actividades tangibles de producción, bajo los parámetros establecidos por los gobernantes, cuyas barreras de entrada son bajas y obtienen bajos rendimientos.

En definitiva, a nivel de planificación de políticas públicas, es necesario entender cómo funcionan hoy las cadenas globales de valor para generar derrames y eslabonamientos desde la exportación y la inversión extranjera directa hacia la economía local. A su vez, la participación en CGV dinámicas y en eslabones de alto valor agregado repercute de manera decisiva en la generación de divisas, empleos calificados y nuevas oportunidades para crear externalidades positivas y encadenamientos dentro de las economías locales.

En contraste con lo que sucedía anteriormente, cuando el comercio de bienes finales se explicaba a partir de las dotaciones de factores y tecnologías estáticas, en la actualidad existe una fragmentación internacional de actividades en contextos marcadamente dinámicos que induce a una creciente especialización en etapas productivas y procesos para abastecer demandas que se tornan universales (Bisang, Anlló y Campi, cita en Kosacoff y Mercado, 2009). Frente a este contexto, una exitosa política de atracción de actividades productivas hacia un país, región o provincia pasará de depender de la creación de ventajas competitivas dinámicas.

\section{Actores subnacionales}

De manera concomitante a estas mutaciones en el ámbito productivo, la trascendencia de los espacios locales se tradujo en el surgimiento de actores subnacionales en el ámbito de las relaciones internacionales. Su participación puso en jaque la concepción realista de la primacía del Estado-Nación, dando cuenta de la transformación compleja de un sistema internacional hacia una sociedad internacional permeada por diferentes actores, principios, ideas e instituciones (Linklater, 1992). Una progresiva necesidad de canalizar las demandas domésticas impulsa a las ciudades, provincias o regiones a participar decididamente en el plano mundial. Sus propósitos no sólo persiguen alcanzar un mayor protagonismo, sino también conseguir objetivos concretos, como ser la captación de recursos externos y la búsqueda de mercados internacionales para la colocación de sus productos materiales y culturales (Zubelzú, 2008). 
Como resultado de la proliferación de interacciones entre actores locales y externos, se ha desarrollado una diversidad de prácticas complementarias a la diplomacia nacional, guiadas por las necesidades e intereses de los diferentes niveles gubernamentales al interior de un Estado. Algunos autores han usado definiciones como diplomacia descentralizada (Aguirre, 2001), diplomacia multinivel (Hocking, 1993), diplomacia constitutiva (Kinkad, 2009), diplomacia federativa (Schiavon, 2010) y diplomacia sub-estatal (Criekemans, 2001), entre otros. En su conjunto, esta multiplicidad de políticas desarrolladas por los entes subnacionales se denomina paradiplomacia.

El término de "paradiplomacia" se encuentra primigeniamente en los escritos de Ivo Duchacek (1986) y representa un marco conceptual adecuado para analizar, encuadrar y explicar este nuevo fenómeno de la participación internacional de las unidades subestatales. En concreto, "la paradiplomacia puede ser definida como la participación de los gobiernos no centrales en las relaciones internacionales, a través del establecimiento de contactos permanentes o ad hoc con entidades públicas o privadas extranjeras, con el propósito de promover diversos aspectos socioeconómicos o culturales" (Cornago, 2001). No obstante, no debe pensarse el ascenso de la paradiplomacia en detrimento de la diplomacia estatal, sino que se trata de un conjunto de interrelaciones transnacionales que se superponen parcialmente a aquélla y complejizan el escenario político mundial (Ferarro, 2005).

Tomando como punto de partida estas definiciones, el presente trabajo buscará echar luz sobre las implicancias y alcances de la paradiplomacia, haciendo foco en las políticas paradiplomáticas económicas que contribuyan no sólo a la inserción local en las cadenas globales de valor, sino también en aquellas acciones que favorezcan el upgrading dentro de ellas.

\section{Prolegómenos de la Paradiplomacia}

En el universo académico de las Relaciones Internacionales, una vez concluida la Primera Guerra Mundial, se consolidó la idea de la centralidad del Estado como unidad indiscutible del sistema internacional. No obstante, las posteriores transformaciones acaecidas durante el siglo XX van a quebrar ese orden. Contrapuestos a las teorías realistas empleadas en el análisis de las relaciones internacionales, Keohane y Nye (1979) son los primeros en exponer, a través de la teoría de la interdependencia, la creciente participación e importancia de los diferentes actores gubernamentales y no gubernamentales, y romper el estatocentrismo vigente hasta entonces en la disciplina académica.

Una de las consecuencias directas de este quiebre teórico es que, durante el transcurso de unas pocas décadas, hemos sido testigos de la pérdida de preeminencia del EstadoNación, que ha cedido poco a poco parte de su soberanía y prerrogativas. ${ }^{6}$ Las ciudades o regiones al interior del Estado-Nación comenzaron a cobrar cada vez mayor autonomía y

\footnotetext{
${ }^{6}$ Huelga decir que no se está planteando la desaparición del Estado. El objetivo aquí buscado apunta a poner en evidencia los cambios que sufrió este, en favor de diversas entidades supra y subnacionales. La primacía de la política exterior central del Estado no está en cuestión, pero estas mutaciones dan pie a la existencia del debate, entre otros, acerca de la creciente importancia de la paradiplomacia y sus efectos colaterales.
} 
presencia en el ámbito internacional, y llegaron a consolidar relaciones económicas, culturales y políticas entre dichos entes subnacionales.

Autores posmodernos, como A. Wendt, R. Cox y R. Ashley -entre otros-, han abierto notablemente la agenda temática de las relaciones internacionales. Este proceso de metamorfosis del escenario internacional explica el surgimiento y desarrollo de la paradiplomacia. Esta es, sin duda, una de las más claras pruebas de la mutación que ha sufrido la comunidad internacional. "La atomización de la estructura del Estado como órgano burocrático administrativo ha generado un vacío de autoridad que permite el surgimiento de entidades sustituto, obligadas a redistribuirse cuotas de poder entre sí a fin de mantener la coherencia interna del Estado como actor del escenario internacional" (Sarquis, 2009. Cita en Coord. Zeraoui, p. 51).

Como se mencionó sucintamente, a mediados de los años setenta surgieron los primeros análisis de este fenómeno a partir de los escritos de Duchacek, aunque se constriñeron principalmente a una descripción de las actividades internacionales de las regiones, más que a un abordaje conceptual del mismo. Hacia finales de la siguiente década, junto con Soldatos (1988), propusieron el término 'paradiplomacia', el cual fue rápidamente aceptado en la academia. Desde entonces, sobrevino una multiplicación de conceptos que presentan variaciones entre sí, algunas más significativas que otras, pero todas hacen referencia al accionar internacional de actores subnacionales.

En aras de evitar galimatías, el presente trabajo empleará como definición de paradiplomacia a la antedicha por Cornago (2001) y resaltará los aspectos que se consideran indispensables. Así, es manifiesta la participación de los gobiernos no centrales en las relaciones internacionales y se descarta el accionar de empresas privadas, universidades, etc. Al adoptar esta definición se resalta la independencia de los mencionados actores respecto de la autoridad nacional ${ }^{7}$ (ver Hocking o Kincaid) y se elimina cualquier entrecruzamiento con la política exterior. Finalmente, si bien se tomará en consideración como actividad paradiplomática el establecimiento de contactos permanentes o ad hoc con entidades públicas o privadas extranjeras; su propósito, para el presente trabajo, se concentrará en la promoción de diversos aspectos económicos, y se dejarán a un lado otro tipo de motivaciones.

Asimismo, desde el fin de la Segunda Guerra Mundial, se asiste a una liberalización creciente del comercio internacional. Ello impulsó a las entidades sub-estatales a desarrollar estrategias a nivel internacional para poder competir con otras entidades, ya sean del mismo país o de otro, en la carrera para atraer a los inversionistas internacionales. Los actores subnacionales se vieron constreñidos a diseñar estrategias más atractivas para los inversionistas, como políticas de promoción de las inversiones y políticas de servicios a los inversionistas, entre muchas otras. Estas pugnas por la localización de inversiones a través del recurso indiscriminado a los incentivos fiscales han derivado en intensas disputas entre entidades subnacionales (ver Ferraro, 2012).

Dentro de la diversidad de acciones que pueden impulsar los actores subnacionales

\footnotetext{
${ }^{7}$ Para un estudio más profundo de los gobiernos no centrales consultar Hocking (2001) y Kincaid (2003).
} 
(financieras ${ }^{8}$, culturales $^{9}$, transfronteriza ${ }^{10}$ y ambientales $^{11}$, entre otras), existe una categoría sobre la que se trabajará aquí y que permite una vinculación directa con el estudio de las CGV. Bajo la clasificación de acciones paradiplomáticas económicas se encuentran, a su vez, una diversidad de actividades y/o políticas públicas. En efecto, durante la siguiente sección se buscará profundizar sobre las políticas paradiplomáticas económicas en general, para luego dar paso a un nuevo subtipo de política subnacional, orientada al upgrading dentro de las CGV.

\section{Paradiplomacia económica}

Paul Kennedy, en Hacia el siglo XXI (1993), menciona que la lógica del mercado global "no presta atención al lugar en el que se hace un producto". Es por ello que argumenta que "el estado-nación y su seguridad también se ven amenazados por la nueva división internacional de la producción y el trabajo (p. 169)." Esta visión economicista se encuentra vinculada a la localización de factores productivos, lo que potencia una nueva geografía económica e incentiva al desarrollo endógeno. Los estudios e investigaciones de la paradiplomacia económica (ver Bueno, 2012; Bessa Maia, 2012) toman como eje a la glocalización de las actividades productivas y colocan el accionar local en el centro de los debates, en aras de potenciar actividades económicas.

Según Keating (cita en Bessa Maia y Sombra Sarabia, 2016, p. 124),

económicamente las regiones buscan inversiones, mercados para sus productos y tecnología para modernizarse. [...] Las inversiones externas directas son medios para generar empleo y crecimiento, así como también orientarse a nuevos sectores económicos, aunque conlleva dependencia e inseguridades ya que el capital escapa tan simple como ingresó. Ello es generalmente balanceado por políticas para crear sectores empresariales locales, especialmente conformado por pequeñas y medianas empresas. Mercados y promoción de las exportaciones son importante para las pequeñas empresas, quienes no disponen de conexiones internacionales, conocimientos o recursos para establecerlos.

En la misma dirección, Lecours (cita en Bessa Maia y Sombra Sarabia, 2016, p. 125) destaca que entre los principales objetivos de la paradiplomacia se encuentran los asuntos económicos.

"En este contexto, los gobiernos subnacionales buscan desarrollar una presencia internacional con el objetivo de atraer inversiones extranjeras y compañías internacionales a la región, así como también identificar nuevos

\footnotetext{
${ }^{8}$ Ver Bessa y Sombra, 2016.

${ }^{9}$ Ver Morales Dábila y Reyes Silva, 2016.

${ }^{10}$ Ver Oddone, 2013.

${ }^{11}$ Ver Rodríguez Vázquez y Lucatello 2013.
} 
mercados para sus exportaciones. [...] Ello es consecuencia directa de la competencia económica global."

Entre las motivaciones económicas, la paradiplomacia puede asumir diferentes modalidades, como puede ser: i) la promoción de las exportaciones o el turismo extranjero (para diplomacia comercial), ii) la captación de recursos financieros externos (paradiplomacia financiera), iii) la atracción de inversiones extranjeras directas (paradiplomacia inversora), y iv) la absorción y trasmisión de conocimientos y tecnología por vía de la cooperación técnica (paradiplomacia cooperativa) (Bessa Maia, 2012).

Es a partir de esta clasificación que se pueden identificar aquellas acciones locales de impacto internacional que estimulan la atracción de grandes inversiones a territorios subnacionales para localizar plantas industriales o grandes emprendimientos en sus territorios. Los representantes estatales emprenden misiones en el extranjero para anunciar al mundo que existen oportunidades de inversiones atractivas en sus estados y que estos brindan apoyo infraestructural, como rutas, autopistas y una mayor aplicación de la ley y el orden (Mattoo y Jacob, 2009 cita en Bessa Maia, 2012).

Es a partir de estos conceptos que hemos arribado a un supuesto subyacente a las motivaciones económicas que es meritorio hacer explícito. La IED es considerada un factor que ayuda al crecimiento y desarrollo económico y, además, es especialmente importante en los países en vías de desarrollo, principalmente porque no atrae únicamente dinero, sino que también, al aumentar los intercambios y la participación de empresas trasnacionales, contribuye a un mejor acceso a nuevas tecnologías. La mayor parte de la literatura coincide en que un flujo entrante de inversión de las multinacionales puede estimular el desarrollo local, al aumentar y mejorar los recursos y capacidades (capital social, tecnología, acceso al mercado), incrementar la competencia, mejorar la asignación de recursos, desarrollar los recursos humanos y crear empleo, entre otras acciones (Lascuráin y Villafuertes, 2018).

Independientemente de las estrategias que los gobiernos nacionales asuman para incentivar la radicación de IED, la necesidad de atraer flujos e inversiones a los territorios se convierte en una actividad política en el interior de los estados, regiones y ciudades para potenciar el crecimiento económico de sus territorios, lo que los lleva a desarrollar diversas tácticas para lograrlo. En efecto, los complejos procesos de negociación entre un país anfitrión y una multinacional han sido estudiados desde los años 60. Mientras las empresas multinacionales buscan maximizar sus beneficios económicos, los gobiernos de los países menos desarrollados buscan maximizar el valor agregado de las actividades que las empresas multinacionales realizan dentro de sus fronteras.

Por su parte, la decisión de una multinacional de radicarse en un determinado país puede ser analizada bajo diversas perspectivas conceptuales, como la teoría de la localización ${ }^{12}$ o la teoría del ciclo de vida del producto ${ }^{13}$. Sin embargo, el paradigma ecléctico de

\footnotetext{
${ }^{12}$ La ubicación de la producción se determina por los recursos que necesita para su actividad. Ver Krugman et al, 2015.

${ }^{13}$ Propone que la ubicación de producción de ciertos tipos de productos cambia a medida que pasan por sus ciclos de vida. Ver Vernon, 1973.
} 
Dunning ${ }^{14}$ proporciona un marco útil para categorizar la mayoría de las investigaciones analíticas y empíricas sobre IED. Si se contempla el foco de análisis de este estudio, las estrategias paradiplomáticas económicas se inscribirían dentro de ventajas para fomentar la atracción de IED hacia una región o ciudad frente a otras alternativas (location).

Otra consecuencia esperable de la globalización económica y la deslocalización productiva es una mayor rivalidad entre las unidades subnacionales en el mercado internacional. En una economía internacional de alta competencia, las regiones buscan generar vínculos e incentivos con actores privados basados en la existencia de intereses mutuos y tienden a diseñar elementos para diferenciarse de otras geografías. De esta manera, se obligan a desarrollar políticas de promoción de inversiones, de servicios a inversionistas, incentivos financieros, y acuerdos de colaboración con otros gobiernos, organizaciones gubernamentales internacionales o no gubernamentales y sector privado. En relación con eso, José Luis Rhui-Sausi (2008, p. 6) precisa que "debido a sus características específicas, los gobiernos locales se han insertado de forma creciente en tareas previamente desarrolladas por los gobiernos nacionales para enfrentar los nuevos desafíos impuestos por la globalización de la economía".

En algunos casos conosureños, estas pugnas por la localización de inversiones a través del uso indiscriminado de los incentivos fiscales han derivado en intensas disputas entre entidades subnacionales, lo cual redundó en un importante desperdicio de recursos fiscales, en beneficio exclusivo de las grandes corporaciones transnacionales (Ferraro, 2005).

Por su parte, la perspectiva de CGV permite enmarcar las diversas fases productivas fragmentadas territorialmente, sus interrelaciones y vínculos con las ciudades y/o regiones donde se radican, y explicar las dinámicas existentes entre éstas y los requerimientos de los mercados internacionales. Sin embargo, como ya se mencionó, no todos los eslabones de una cadena productiva repercuten de la misma manera en la economía local.

En consecuencia, para maximizar los beneficios de las economías domésticas, las políticas paradiplomáticas deberían ser estructuradas en base a dos principios. Por un lado, deben motivar la localización en sus territorios de eslabones productivos de una CGV. Ello se encontraría acorde a las premisas impulsadas por los organismos internacionales y fomentaría el desarrollo económico local. Sin embargo, si este fuera el único principio rector de las políticas subestatales, se omitirían los aportes neoshumpeterianos, que identifican asimetrías entre las diversas actividades productivas deslocalizadas. En consecuencia, otro principio basal que deberían incorporar los actores subnacionales sería la promoción de innovaciones tecnológicas que permitan el escalamiento hacia actividades más complejas dentro de la cadena global de valor. Dichas actividades de mayor valor agregado suelen caracterizarse por altas barreras de entrada y, generalmente, son retenidas por las empresas de países desarrollados. Por el contrario, en los países en desarrollo, las empresas tienden a ser bloqueadas en las actividades tangibles de producción.

En resumen, los actores subnacionales no sólo deben incentivar la radicación de eslabones productivos de las CGV, sino también deben estimular la superación de las barreras

\footnotetext{
${ }^{14}$ Llamado OLI a partir de sus siglas en inglés: ownership (propiedad), location (ubicación) e internalization (internalización).
} 
de entradas hacia eslabones más sofisticados y con mayores rentas. De este modo, se propone elaborar políticas paradiplomáticas orientadas al upgrading que tiendan a la creación de ventajas competitivas dinámicas locales, con la consecuente producción de mayores beneficios para sus sociedades.

\section{Paradiplomacia orientada al upgrading}

La literatura existente sobre políticas paradiplomáticas económicas resalta la promoción de los territorios como destinos atractivos para la radicación de inversiones extranjeras directas. Para lograrlo, las unidades subnacionales se valieron de misiones de promoción y el establecimiento de incentivos para los inversores, como ser la exención de impuestos, las garantías de préstamos y el reintegro de inversión, entre otros. Sin embargo, estas acciones en materia económica presentan un patrón común para la radicación de inversiones, con la presunción de que cualquier tipo de inversión redunda en beneficios económicos locales. La nueva internacionalización productiva que tiene lugar a nivel mundial habilita la posibilidad de focalizarse en aquellas actividades que potencien un crecimiento de largo plazo, basado en incrementos genuinos y sistemáticos de la productividad y la competitividad, generando derrames en la economía local.

Las políticas internacionales locales implementadas no maximizaron los beneficios derivados de las transformaciones de la configuración productiva mundial, o al menos no se diseñaron en pos de ello. Por consiguiente, se identifica un área de vacancia cognoscitiva dentro de la paradiplomacia económica, vinculada a las gestiones subnacionales que promuevan la localización de eslabones productivos de alto impacto en sus geografías, así como estrategias de upgrading dentro de las mismas, con el objetivo de maximizar la participación en dichas cadenas y obtener mayores rentas.

Un concepto vital dentro de la perspectiva de CGV, al cual las actividades paradiplomáticas podrían orientarse, es el upgrading. Este hace referencia a todas aquellas acciones que promueven la realización de mejores productos, métodos para hacerlos de manera más eficiente o avances que permitan la incorporación de actividades más calificadas. Los estudios sobre cadenas globales de valor hacen hincapié en la posibilidad de los productores de países en desarrollo de adquirir nuevas habilidades y conocimientos, los cuales pueden ser estimulados a través de la participación en las CGV.

El análisis de cadena de valor puede ayudar a explicar la creciente disyuntiva entre el despliegue global de las actividades y los ingresos, particularmente desde una perspectiva dinámica. La renta es la utilidad o el beneficio que se obtiene de una actividad económica, y los mayores resultados se acumulan en aquellas partes que se protegen de la competencia e involucran barreras de entrada.

Partiendo de esta noción, la perspectiva de CGV permite inicialmente realizar un mapeo de las actividades, lo cual facilita la capacidad de descomponer la rentabilidad de toda la cadena de valor en las recompensas alcanzadas por los diferentes eslabones que la conforman. En segunda instancia, analiza la manera en que firmas particulares, ciudades y regiones están enlazados a una economía global. Y en tercera medida, por la concentración en las instituciones que dirigen la especialización internacional, el análisis de cadena de valor 
identifica la palanca normativa que puede ser usada para alterar esos patrones de distribución (Kaplinsky y Morris, 2001).

Los procesos de upgrading, al generar una producción con un mayor valor agregado, permiten alcanzar nuevas rentas y modificar las estructuras de las CGV. Ello implica una superación respecto del concepto estático de Ricardo de la ventaja comparativa, dado que el éxito en la mejora a nivel de empresa permite la adquisición dinámica de competitividad en nuevos nichos de mercado, sectores o fases de la cadena productiva (Lall, 2001; Pietrobelli, 1997). En palabras de Kaplinsky (2001), se puede sostener que "las cadenas de valor son depósitos para la renta, y esas rentas son dinámicas."

Un punto sensible es identificar qué actividades y tecnologías de una firma se mantienen en las casas centrales de las empresas trasnacionales y cuáles pueden ser subcontratadas a otras empresas. Las estructuras de gobierno de las CGV crean barreras para el upgrading local. No obstante, esas barreras se pueden superar dependiendo del cuán estrecho sea el control en la cadena y de la habilidad de las empresas y de los recursos locales para mejorar. Es aquí donde la paradiplomacia económica desempeña un rol destacado en el acompañamiento de estos procesos de upgrading local.

Según Albuquerque (2004), la calidad de la inserción internacional de un espacio subnacional está fuertemente vinculada a su capacidad de producción y generación de ventajas competitivas dinámicas. En la misma dirección y en palabras de Porter (2007), "las industrias competitivas de un país están usualmente vinculadas a una ciudad o región, dando lugar al concepto de ventaja competitiva local".

La creación a nivel local de estas condiciones favorables exige aplicar políticas selectivas a partir de la identificación de sectores productivos con alto potencial de valor agregado. Otro aspecto relevante a considerar es que cada cadena productiva responde a diferentes intereses y motivaciones, lo que debe traducirse en que las políticas para su seducción sean específicas y focalizadas. Las actividades económicas dinámicas, caracterizadas por la innovación y las complementariedades institucionales, dan lugar a la diversificación de las exportaciones basadas en la inversión hacia nuevas actividades con mayor valor agregado y contenido tecnológico.

\section{Conclusiones}

La transnacionalización de la economía es resultado de una producción a escala global, caracterizada por una creciente fragmentación de los procesos productivos en diversas localizaciones que maximizan sus beneficios. Las investigaciones sobre las cadenas globales de valor examinan las diferentes formas en las que se integran y se relacionan los sistemas de producción y distribución mundial, así como las posibilidades de las empresas en los países en desarrollo para mejorar su posición en los mercados mundiales.

Esta segmentación internacional de los procesos productivos y la consecuente conformación de sistemas globales de producción han generado nuevos desafíos para las ciudades y regiones, ávidas de identificar tanto las oportunidades como los obstáculos para mejorar las capacidades locales. La comprensión respecto de las gestiones de las CGV ayuda a 
discernir la distribución de las rentas a lo largo de una cadena, así como las oportunidades de upgrading para mejorar la posición relativa de los actores vernáculos dentro de ellas.

Como testigos de los modos de producción vigentes en la actualidad, es plausible sostener que el desarrollo local a largo plazo requiere de políticas paradiplomáticas que se caractericen por una apropiada planificación que identifique sectores estratégicos y articulaciones, que estén centradas en la manufactura de media y alta tecnología, intensivas en diseño e innovación, así como también en los servicios basados en conocimiento. La participación en CGV permite el acceso a nuevas tecnologías, habilidades empresariales y redes de innovación que tienden a incrementar la productividad y deberían mejorar la calidad del empleo y de los salarios (Oddone, 2016a). En consecuencia, se deben incentivar actividades productivas locales que se distancien de aquellas manos de obra intensivas y se concentren en actividades intangibles, las cuales requieren de recursos humanos calificados.

En concordancia con López y Ramos (cita en Kosacoff y Mercado 2009, p.86), es evidente el potencial que pueden tener las empresas trasnacionales para influir sobre la configuración productiva de los países en desarrollo, no sólo a través de la presencia directa sino especialmente a través del vínculo con los proveedores y clientes que forman parte de las CGV. Siendo así, pasan a ser centrales no sólo las políticas de atracción de inversiones sino también las acciones de estímulo al desarrollo de capacidades de absorción doméstica, la mejora de los bienes públicos, el desarrollo de activos específicos y la promoción del involucramiento de las ET [empresas trasnacionales] con el medio local, entre otras.

El nuevo paradigma de producción global impone una agenda nueva a las autoridades locales en materia de políticas paradiplomáticas. La ausencia de políticas públicas articuladas, destinadas a fomentar la participación local en las CGV, que impulsen el desarrollo de un ecosistema de interacción entre los agentes, mejoren las capacidades de absorción doméstica y promuevan el upgrading hacia actividades de alto valor agregado en sus geografías, representa un punto de partida para los gobiernos locales.

\section{Bibliografía}

Aguirre, I. (2001). ¿Qué sentido tiene hablar de paradiplomacia? Una encuesta intertextual entorno a un neologismo polisémico. En F. Aldecoa y M. Keating (eds.). Paradiplomacia: las relaciones internacionales de las regiones. Marcial Pons: Madrid.

Alburquerque, F. (2004). El enfoque del desarrollo económico local. Cuaderno de capacitación No. 1. Serie: Desarrollo Económico Local y Empleabilidad Programa AREA - OIT en Argentina - Italia Lavoro Buenos Aires, Organización Internacional del Trabajo.

Bessa Maia, J. N. (2012). A Paradiplomacia Financeira dos Estados Brasileiros: evolução, fatores determinantes, impactos e perspectivas. Tese de Doutorado. Instituto de Relações Internacionais. Universidade de Brasilia, Brasilia.

Botto, M. y Scardamaglia, V. (2011) Creando capacidades: las negociaciones económicas 
internacionales y las provincias argentinas. ¿Un rol para el sector privado?" Serie Aportes №15. ISSN 1853-1113. Buenos Aires

Bueno, I. (2012). Paradiplomacia Económica. Trajetórias e Tendências da Atuação Internacional dos Governos Estaduais do Brasil e dos Estados Unidos. Editora Verdana: Brasília.

Buzan, B. (1993). From International System to International Society: Structural Realism and Regime Theory meet the English School. International Organization no 47.

Carreón, P. (2007). Paradiplomacia y su desarrollo en el mundo. Protocolo Foreing Affairs and Lifestyle. Sección Internacional. México.

Colacrai, M y Zubelzú, G. (2004). Las vinculaciones externas y la capacidad de gestión internacional desplegadas por las provincias argentinas en la última década. Una lectura desde las relaciones internacionales. CARI: Buenos Aires.

Cornago, N. (2001). Diplomacia, paradiplomacia y redefinición de la seguridad mundial: dimensiones de conflicto y cooperación. En F. Aldecoa y M. Keating (eds.). Paradiplomacia: las relaciones internacionales de las regiones. Marcial Pons: Madrid.

Cornago, N. (2001). La descentralización como elemento de innovación diplomática: aproximación a sus causas estructurales y lógicas de acción. En L. Maira (ed.). La política internacional subnacional en América Latina. Libros del Zorzal: Buenos Aires.

Costero Garbarino, M. C. (2013). Paradiplomacia en México. La actividad exterior del estado de San Luis Potosí. Revista de El Colegio de San Luis. Nueva época. Año V, número 9.

Criekemans, D. (2001). Are the boundaries between paradiplomacy and diplomacy watering down? Preliminary findings and hypotheses from a comparative study of some regions with legislative power and small states. 2nd Global International Studies Conference, World International Studies Committee (WISC). Slovenia.

Duchacek, I. (1986). The territorial dimension of politics: within, among and across nations. Westview Press. London.

Felli. L. (2015). La paradiplomacia en la revista Relaciones Internacionales. Revista Estudios №49. ISSN: 1852-1568

Ferraro, D. (2012). La acción internacional de los gobiernos locales. Montevideo Internacional. Disponible en: http://www.paradiplomacia.org/upload/downloads/12f9598dc45f6299fb08c15afc00c44emvd_internacional.pdf

Ferrero, M. (2005). La globalización en acción: regionalización y paradiplomacia en Argentina y el Cono Sur latino americano, ponencia presentada en el VII Congreso Nacional de Ciencia Política, organizado por la Sociedad Argentina de Análisis Político (SAAP) “Agendas regionales en escenarios de conflicto”, Córdoba (Argentina), 15-18 de noviembre.

Gereffi, G.; Humphrey, J. y Sturgeon, T. (2005). The governance of global value chains. Institute of Development Studies. Review of International Political Economy 12:1 February 2005: 78-104. Disponible en: http://www.fao.org/fileadmin/user_upload/fisheries/docs/GVC_Governance.pdf

Hernandéz Sampieri, R., Collado, C., y Baptista Lucio, P. (2006). Metodología de la Investigación. 4ta ed. Mc Grall Hill Interamericana: México. 
Hocking, B. (1993). Foreign Relations and Federal States. Leicester University Press: Londres.

Hocking, B. (2000). Vigilando la frontera: globalización, localización y capacidad de actuación de los gobiernos no centrales. En Felli. L. La paradiplomacia en la revista Relaciones Internacionales. Revista Estudios No49. 2015. ISSN: 1852-1568

Kaplinsky, R. (2000). Spreading the gains from globalization. IDS Working Paper 110. Disponible en: https://www.ids.ac.uk/files/Wp110.pdf

Kaplinsky, R. y Morris, M. (2001). A Handbook for Value Chain Research. Vol. 113. IDRC. [Disponible en: http://www.srp-guinee.org/download/valuechain-handbook.pdf

Keating, M. (2001). Regiones y asuntos internacionales: motivos, oportunidades y estrategias. En F. Aldecoa y M. Keating (eds.). Paradiplomacia: las relaciones internacionales de las regiones. Marcial Pons: Madrid.

Kennedy, P. (1993). Hacia el Siglo XXI. Plaza \& Janés: Barcelona..

Keohane, R. y Nye, J. (1988). Poder e Interdependencia. La Política Mundial en Transición. GEL Editores: Buenos Aires.

Kinkad, J. (2009). Bibliographic Resources for Federalism and Foreign Affairs. En H. Michelmann (ed.). Foreign Relations in Federal Countries. Forum of Federations y McGill-Queen's University Press. Booklet Series, vol. 5. Montreal.

Lall, S., Albaladejo, M. y Zhang, J. (2004). Mapping Fragmentation: Electronics and Automobiles in East Asia and Latin America. Oxford Development Studies 32, no. 3. Disponible en: http://economics.ouls.ox.ac.uk/12780/1/qehwps115.pdf

Lascurain Fernández, M. (2016). Challenges of economic globalization. Revista de Relaciones Internacionales. Estrategia y Seguridad. 12 (1), pp. 23-50.

Lascuráin Fernández, M. y Villafuertes, F. (2018). Multinationals and the bargaining dynamic among Least Developed Countries. Brazilian Journal of International Relations, V. 7, N. 1.

Linklater, A. (1992). The Question of the Next Stage in International Relations Theory: A Critical-Theoretical Point of View. Millennium, 21(1), 77-98.

DOI: https://doi.org/10.1177/03058298920210010601

López, A. y Ramos, D. (2009). Inversión Extranjera Directa y Cadenas Globales de Valor.En Bernardo Kosacoff, (eds.). La Argentina ante la nueva internacionalización de la producción. CEPAL. Disponible en: http://www.cepal.org/argentina/noticias/noticias/5/37995/libro_CEPAL_PNUD.pdf

Maira, L. (2010). La Política Internacional Subnacional en América Latina. El Zorzal: Buenos Aires.

Martín, M. y Oddone. N. (2010). Las ciudades y los poderes locales en las Relaciones Internacionales Contemporáneas. Observatorio Iberoamericano del Desarrollo Local y la Economía Social: Granada.

Natalizio, V. (2007). La Multicausalidad y Multidimensionalidad de la Acción Internacional de los Actores Subnacionales Argentinos. El caso de la Ciudad Autónoma de Buenos Aires. Centro Argentino de Estudios Internacionales: Buenos Aires 
Oddone, N. (2016a). Política comercial y cadenas de valor en Argentina contemporánea: ¿una nueva etapa? PUENTES, Volumen 17, Número 2.

. (2016b). Cadenas de valor en la Argentina: notas para una política comercial inteligente. Centro de Estudios Internacionales Contemporáneos: Córdoba.

Oddone, N. y Padilla Pérez, R. (2014). El escalamiento en las cadenas de valor a través de servicios profesionales y de soporte. Puentes. Volumen 15, Número 3.

Oszlak, O. (1997). Estado y Sociedad: ¿Nuevas reglas de juego? Reforma y Democracia. CLAD, No. 9.

Padilla Pérez, R. (2014). Fortalecimiento de las cadenas de valor como instrumento de la política industrial. Metodología y experiencia de la CEPAL en Centroamérica. Desarrollo Económico. Comisión Económica para América Latina y el Caribe (CEPAL). Disponible en: http://www.cepal.org/es/publicaciones/36743-fortalecimiento-cadenas-valor-como-instrumento-la-politica-industrial

Porter, M. (2007). La ventaja competitiva de las Naciones. Harvard Business Review, ISSN 0717-9952, Vol. 85, №. 11, 2007, pp. 69-95

Rhi-Sausi, J.L. (2008). La cooperación internacional en los procesos de descentralización y regionalización de los países latinoamericanos. La experiencia Italia-región de Atacama. Revista OIDLES. Vol 2, № 3.

Robertson, R. (1995). Glocalization: Time-Space and Homogeneity-Heterogeneity. En Mike Featherstone, Scott Lash and Roland Robertson. Global Modernities. Sage: London.

Sarquís, D. (2009). Fundamentos teóricos para la reflexión en torno a la paradiplomacia. En Zeraoui, Z. (coord..). Regionalización y paradiplomacia: La política internacional de las regiones. Montiel \&Soriano Editores S.A.: México.

Schiavon, J. (2010). Las relaciones exteriores de los gobiernos estatales: El caso de México. En Maira, L. (ed.). La política internacional subnacional en América Latina. Libros del Zorzal. Buenos Aires.

Siles, M. (2014). La paradiplomacia de las provincias argentinas y la responsabilidad internacional derivada de su actuación. Ponencia en el VII Congreso de Relaciones Internacionales del Instituto de Relaciones Internacionales de la U.N.L.P. La Plata.

The Global Value Chains Initiative. Disponible en: www.globalvaluechains.org.

Universidad del Rosario. (2016). Dossier Temático: La paradiplomacia y la política internacional de las regiones. Desafíos. Vol. 28, Núm. ISSN-e: 2145-5112, ISSN: 01244035, Colombia.

Zeraoui, Z. (Coord.). 2013. Teoría y práctica de la Paradiplomacia. Montiel \&Soriano Editores S.A.: México.

Zubelzú, G. (2008). Los gobiernos subnacionales en el escenario internacional. En Iglesias, E. (dir.) Las provincias argentinas en el escenario internacional. CARI: Buenos Aires. 